\title{
Preparation of methoxyazaisoindolinones
}

\author{
Andrzej Jóźwiak and Aleksandra K. Szcześniak \\ Department of Organic Chemistry, University of Łódź, 90-136 Łódź, Narutowicza 68, Poland \\ E-mail: ajozwiak@uni.lodz.pl,olaszcz@,uni.lodz.pl
}

Dedicated to Professor Jan Epsztajn on the occasion of his $75^{\text {th }}$ birthday

\begin{abstract}
Preparation of methoxyazaisoindolinones $\mathbf{3}$ and $\mathbf{4}$ via the reaction of hydroxyazaisoindolinones $\mathbf{1}$ and $\mathbf{2}$ with potassium tert-butoxide and iodomethane in $\mathrm{N}, \mathrm{N}$-dimethylformamide is described.
\end{abstract}

Keywords: Azaisoindolinones, Williamson synthesis

\section{Introduction}

3-Alkoxyphthalimidines have been used among others for preparation of compounds modulating the muscarinic $\mathrm{M}_{2}$-receptor and for construction of heterocyclic systems. ${ }^{1}$ It is known that a reaction of $N$-acyliminium ions generated from 3-hydroxyphthalimidines ${ }^{2}$ and their 7-hydroxypyrrolo[3,4-c]pyridine analogs ${ }^{3 a} \mathbf{A}$ with primary and secondary alcohols gave alkoxy derivatives in high yields. Much effort has been devoted to the investigation of 7-substituted 6,7-dihydro$5 H$-pyrrolo[3,4-b]pyridin-5-ones as cyclic nicotinamide analogues or aza-analogues of phthalimidines, some of which are known to be biologically active compounds. ${ }^{3}$ In contrast, in the literature we have not found any information but one ${ }^{3 \mathrm{~d}}$ concerning alkoxyazaisoindolinones of type B. In this paper we described useful method for their preparation.

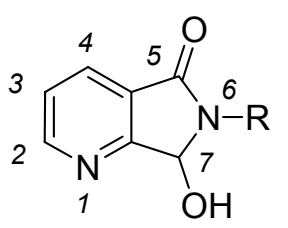

A<smiles></smiles>

B
$X=N, Y=C H$
$X=C H, Y=N$ 


\section{Results and Discussion}

Application of $N$-acyliminium ion strategy $\left(\mathrm{MeOH}, \mathrm{MeSO}_{3} \mathrm{H}\right.$ or $\left.\mathrm{H}_{2} \mathrm{SO}_{4}\right)$ in the case of 6,7dihydro-5-hydroxy-6-aryl-5H-pyrrolo[3,4-b]pyridin-7-ones 1 and 2,3-dihydro-3-hydroxy-2-aryl$1 H$-pyrrolo[3,4-c]pyridin-1-ones $\mathbf{2}$ unexpectedly resulted in very low yields of desired methoxyproducts 3 or 4 (less than 10\%). Therefore, we focused our attention on Williamson synthesis as an alternative way for the preparation of the methoxy derivatives 3 and $\mathbf{4}$. On the basis of such viewpoints, the compounds $\mathbf{1}$ and $\mathbf{2}$ were converted into the corresponding methoxyazaisoindolinones by reaction with potassium tert-butoxide and next iodomethane.

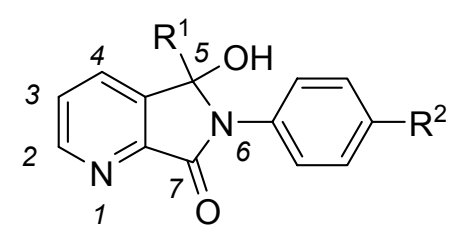

1

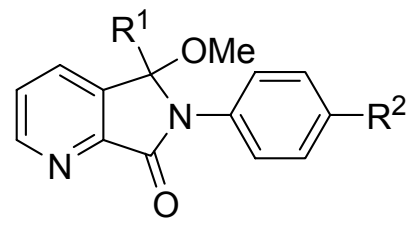

3

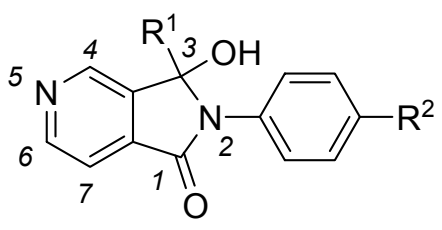

2

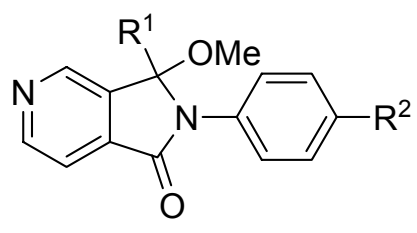

4
a: $\mathrm{R}^{1}=\mathrm{R}^{2}=\mathrm{H}$;
b: $\mathrm{R}^{1}=\mathrm{H}, \mathrm{R}^{2}=\mathrm{OMe}$
c: $R^{1}=P h, R^{2}=H$,

In all cases, iodomethane appeared to be insignificantly active towards $N$-alkylation of the pyridine nucleus (under the conditions used). The described methodology represents an effective route for the preparation of methoxyazaisoindolinones 3 and 4.

\section{Experimental Section}

General Procedures. Melting points were determined using a Boetius hot-stage apparatus and they are uncorrected. IR spectra were recorded on a Zeiss-Jena Specord 71-IR. H NMR spectra were determined at $200 \mathrm{MHz}$ on a Varian Gemini 200 using TMS as an internal standard. Analytical thin layer chromatography tests (TLC) were carried out on aluminium oxide (Merck, $60 \mathrm{~F}_{254}$ neutral, layer thickness $0.2 \mathrm{~mm}$ ) or silica gel (Merck, $60 \mathrm{~F}_{254}$, layer thickness $0.2 \mathrm{~mm}$ ). Column chromatography separations were performed on aluminium oxide (90 active, basic, 
Merck 0.063-0.200 mm/70-230 mesh ATSM) or silica gel (Macherey Nagel \& Co. 0.075-0.150 mm/100-200 mesh ATSM). Potassium tert-butoxide (Fluka) were used without further purification. $\mathrm{N}, \mathrm{N}$-Dimethylformamide used was dried by azeotropic distillation with anhydrous benzene, shaken with activated neutral alumina (Brockmann I), filtered, then distilled under argon at reduced pressure and stored over type 4A molecular sieves. ${ }^{6}$ Hydroxyazaisoindolinones $\mathbf{1}$ and $\mathbf{2}$ were obtained according to known procedures: 6,7-Dihydro-5-hydroxy-6-phenyl-5 $\boldsymbol{H}$ pyrrolo[3,4-b]pyridin-7-one (1a), (62\%) m. p. 236-238 ${ }^{\circ} \mathrm{C}$ (methanol), (lit. ${ }^{5 \mathrm{a}} \mathrm{m} . \mathrm{p} \cdot 237-239^{\circ} \mathrm{C}$ ); 6,7-Dihydro-5-hydroxy-6-(4-methoxyphenyl)-5H-pyrrolo[3,4-b]pyridin-7-one (1b), (62\%) m. p. $236-238^{\circ} \mathrm{C}$ (methanol), (lit. ${ }^{5 \mathrm{c}}$ m. p. $\left.237-239^{\circ} \mathrm{C}\right)$; 6,7-Dihydro-5-hydroxy-5,6-diphenyl-5Hpyrrolo[3,4-b]pyridin-7-one (1c), $(65 \%)$ m. p. $162-164^{\circ} \mathrm{C}$ (acetone : hexane - 1:1), (lit. ${ }^{5 b} \mathrm{~m} . \mathrm{p}$. $\left.163-164^{\circ} \mathrm{C}\right)$; 2,3-Dihydro-3-hydroxy-2-phenyl-1 $\boldsymbol{H}$-pyrrolo[3,4-c]pyridin-1-one (2a), (72\%) $\mathrm{m}$. p. $223-225^{\circ} \mathrm{C}$ (acetone), (lit. ${ }^{5 \mathrm{a}} \mathrm{m}$. p. $223-225^{\circ} \mathrm{C}$ ); 2,3-Dihydro-3-hydroxy-2-(4-methoxyphenyl)1H-pyrrolo[3,4-c]pyridin-1-one (2b), (84\%) m. p. 261-263 ${ }^{\circ} \mathrm{C}$ (methanol), (lit. ${ }^{6 \mathrm{c}} \mathrm{m} . \mathrm{p} .260-$ $\left.262^{\circ} \mathrm{C}\right)$; 2,3-Dihydro-3-hydroxy-2,3-diphenyl-1 $\boldsymbol{H}$-pyrrolo[3,4-c]pyridin-1-one (2c), (65\%) $\mathrm{m}$. p. $164-166^{\circ} \mathrm{C}$ (methanol), (lit. ${ }^{5 b}$ m. p. $165-167^{\circ} \mathrm{C}$ ).

\section{Conversion of hydroxyazaisoindolinones 1 and 2 in to methoxyazaisoindolinones 3 and 4}

Potassium tert-butoxide $(0.02$ mole $)$ was added to the stirred mixture of compound $\mathbf{1}$ or $2(0.01$ mole) in $N, N$-dimethylformamide $(20 \mathrm{ml})$ at $0^{\circ} \mathrm{C}$. To the obtained solution iodomethane $(0.05$ mole) was added and the mixture was kept at $0^{\circ} \mathrm{C}$ for $2.5 \mathrm{~h}$. Excess of iodomethane and the solvent was removed under reduced pressure to give a sticky residue. The product was extracted with dichloromethane $(4 \times 15 \mathrm{ml})$ and separated by column chromatography. Compounds 3 and 4 were purified by crystallisation.

The solvent for crystallisation, the isolated yields and the physical and spectral characterisation of products 3 and 4 are given below.

6,7-Dihydro-5-methoxy-6-phenyl-5H-pyrrolo[3,4-b]pyridin-7-one (3a). (56\%), (silica gel, eluent dichloroethane : ethyl acetate - $1: 1, \mathrm{R}_{\mathrm{f}} 0.3$ ), m. p. $142-145^{\circ} \mathrm{C}$ (ethyl acetate); (Found: $\mathrm{C}$, 70.1; H, 5.0; N, 11.4. Calc. for $\mathrm{C}_{14} \mathrm{H}_{12} \mathrm{~N}_{2} \mathrm{O}_{2}$ : C, 69.99; H, 5.03; N, 11.66\%); IR (KBr) $1700 \mathrm{~cm}^{-1}$ $(\mathrm{C}=\mathrm{O}) ;{ }^{1} \mathrm{H}$ NMR $\left(\mathrm{CDCl}_{3}\right) 8.88(1 \mathrm{H}, \mathrm{dd}, \mathrm{J}=4.9$ and $1.3 \mathrm{~Hz}, 2-\mathrm{H}), 8.00(1 \mathrm{H}, \mathrm{dd}, \mathrm{J}=7.7$ and 1.3 $\mathrm{Hz}, 4-\mathrm{H}), 7.87-7.77(2 \mathrm{H}, \mathrm{m}, 2 \mathrm{Ph}$ and $6 \mathrm{Ph}-\mathrm{H}), 7.57(1 \mathrm{H}, \mathrm{dd}, \mathrm{J}=7.7$ and $4.9 \mathrm{~Hz}, 3-\mathrm{H}), 7.51-7.38$ $(2 \mathrm{H}, \mathrm{m}, 3 \mathrm{Ph}$ and $5 \mathrm{Ph}-\mathrm{H}), 7.31-7.20(1 \mathrm{H}, \mathrm{m}, 4 \mathrm{Ph}-\mathrm{H}), 6.51(1 \mathrm{H}, \mathrm{s}, 5-\mathrm{H}), 2.93(3 \mathrm{H}, \mathrm{s}, \mathrm{OMe}) ;{ }^{13} \mathrm{C}$ NMR $\left(\mathrm{CDCl}_{3}\right)$ 164.52, 152.88, 150.75, 136.66, 133.95, 131.81, 129.11, 126.36, 125.77, 121.69, $85.25,49.59$.

6,7-Dihydro-5-methoxy-6-(4-methoxyphenyl)-5H-pyrrolo[3,4-b]pyridin-7-one (3b). (52\%), (aluminium oxide, eluent dichloromethane, $\mathrm{R}_{\mathrm{f}} 0.4$ ), m. p. $108-109^{\circ} \mathrm{C}$ (ethyl acetate : heptane -2 :1); (Found: C, 66.4; H, 5.3; N, 10.4. Calc. for $\mathrm{C}_{15} \mathrm{H}_{14} \mathrm{~N}_{2} \mathrm{O}_{3}$ : C, 66.66; H, 5.22; N, 10.36\%); IR $(\mathrm{KBr}) 1700 \mathrm{~cm}^{-1}(\mathrm{C}=\mathrm{O}) ;{ }^{1} \mathrm{H} \mathrm{NMR}\left(\mathrm{CDCl}_{3}\right) 8.88(1 \mathrm{H}, \mathrm{dd}, \mathrm{J}=4.8$ and $1.4 \mathrm{~Hz}, 2-\mathrm{H}), 7.98(1 \mathrm{H}$, dd, $\mathrm{J}=7.7$ and $1.4 \mathrm{~Hz}, 4-\mathrm{H}), 7.73-7.61(2 \mathrm{H}, \mathrm{m}, 2 \mathrm{Ar}$ and $6 \mathrm{Ar}-\mathrm{H}), 7.56(1 \mathrm{H}$, dd, $\mathrm{J}=7.7$ and $4.8 \mathrm{~Hz}, 3-$ $\mathrm{H})$, 7.05-6.92 (2H, m, 3Ar and 5Ar-H), 6.41 (1H, s, 5-H), $3.83(3 \mathrm{H}, \mathrm{s}, \mathrm{OMe}), 2.96(3 \mathrm{H}, \mathrm{s}, \mathrm{OMe})$; 
${ }^{13} \mathrm{C} \mathrm{NMR}\left(\mathrm{CDCl}_{3}\right) 164.66,157.72,152.93,151.10,134.05,131.78,129.59,126.24,123.92$, 114.50, 85.82, 55.47, 49.87.

6,7-Dihydro-5-methoxy-5,6-diphenyl-5H-pyrrolo[3,4-b]pyridin-7-one (3c). (56\%), (silica gel, eluent dichloroethane : ethyl acetate $-1: 1, \mathrm{R}_{\mathrm{f}} 0.5$ ), m. p. $199-201{ }^{\circ} \mathrm{C}$ (ethyl acetate : heptane -2 : 1); (Found: C, 75.6; H, 5.3; N, 8.8. Calc. for $\mathrm{C}_{20} \mathrm{H}_{16} \mathrm{~N}_{2} \mathrm{O}_{2}$ : C, 75.93; H, 5.10; N, 8.85\%); IR $(\mathrm{KBr}) 1720 \mathrm{~cm}^{-1}(\mathrm{C}=\mathrm{O}) ;{ }^{1} \mathrm{H}$ NMR $\left(\mathrm{CDCl}_{3}\right) 8.85(1 \mathrm{H}, \mathrm{dd}, \mathrm{J}=4.7$ and $1.5 \mathrm{~Hz}, 2-\mathrm{H}), 7.64-7.04$ $(12 \mathrm{H}, \mathrm{m}, \mathrm{Ph}, 3-\mathrm{H}$ and $4-\mathrm{H}), 3.18(3 \mathrm{H}, \mathrm{s}, \mathrm{OMe}) ;{ }^{13} \mathrm{C} \mathrm{NMR}\left(\mathrm{CDCl}_{3}\right)$ 165.55, 152.64, 149.47, 139.29, 137.76, 135.87, 131.25, 128.71, 128.59, 128.26, 126.79, 126.07, 123.86, 95.31, 50.59.

2,3-Dihydro-3-methoxy-2-phenyl-1 H-pyrrolo[3,4-c]pyridin-1-one (4a). (58\%), (aluminium oxide, basic, eluent dichloromethane, $\mathrm{R}_{\mathrm{f}} 0.6$ ), m. p. $69-71^{\circ} \mathrm{C}$ (heptane : ethyl ether - $8: 2$ ); (Found: C, 70.2; H, 5.1; N, 11.7. Calc. for $\mathrm{C}_{14} \mathrm{H}_{12} \mathrm{~N}_{2} \mathrm{O}_{2}$ : C, 69.99; H, 5.03; N, 11.66\%); IR (KBr) $1710 \mathrm{~cm}^{-1}(\mathrm{C}=\mathrm{O}) ;{ }^{1} \mathrm{H}$ NMR $\left(\mathrm{CDCl}_{3}\right) 8.99(1 \mathrm{H}, \mathrm{s}, 4-\mathrm{H}), 8.92(1 \mathrm{H}, \mathrm{d}, \mathrm{J}=4.8 \mathrm{~Hz}, 6-\mathrm{H}), 7.87-7.73$ $(3 \mathrm{H}, \mathrm{m}, 2 \mathrm{Ph}, 6 \mathrm{Ph}$ and 7-H), 7.55-7.40 (2H, m, 3Ph and 5Ph-H), 7.35-7.22 (1H, m, 4Ph-H), 6.60 $(1 \mathrm{H}, \mathrm{s}, 3-\mathrm{H}), 2.98(3 \mathrm{H}, \mathrm{s}, \mathrm{OMe}) ;{ }^{13} \mathrm{C} \mathrm{NMR}\left(\mathrm{CDCl}_{3}\right)$ 164.74, 151.76, 145.71, 140.51, 136.45, $134.05,129.27,126.15,122.06,117.60,86.94,49.93$.

2,3-Dihydro-3-methoxy-2-(4-methoxyphenyl)-1 $\boldsymbol{H}$-pyrrolo[3,4-c]pyridin-1-one (4b). (55\%), (silica gel, eluent dichloroethane : ethyl acetate - $1: 1, \mathrm{R}_{\mathrm{f}} 0.2$ ), m. p. $141-145^{\circ} \mathrm{C}$ (ethyl acetate); (Found: C, 66.7; H, 5.0; N, 10.4. Calc. for $\mathrm{C}_{15} \mathrm{H}_{14} \mathrm{~N}_{2} \mathrm{O}_{3}$ : C, 66.66; H, 5.22; N, 10.36\%); IR (KBr) $1710 \mathrm{~cm}^{-1}(\mathrm{C}=\mathrm{O}) ;{ }^{1} \mathrm{H} \mathrm{NMR}\left(\mathrm{CDCl}_{3}\right) 8.96(1 \mathrm{H}, \mathrm{s}, 4-\mathrm{H}), 8.91(1 \mathrm{H}, \mathrm{d}, \mathrm{J}=4.8 \mathrm{~Hz}, 6-\mathrm{H}), 7.81(1 \mathrm{H}$, $\mathrm{d}, \mathrm{J}=4.8 \mathrm{~J}, 7-\mathrm{H}), 7.68-7.54(2 \mathrm{H}, \mathrm{m}, 2 \mathrm{Ar}$ and 6Ar-H), 7.06-6.92 (2H, m, 3Ar and 5Ar-H), 6.49 $(1 \mathrm{H}, \mathrm{s}, 3-\mathrm{H}), 3.84(3 \mathrm{H}, \mathrm{s}, \mathrm{OMe}), 3.00(3 \mathrm{H}, \mathrm{s}, \mathrm{OMe}) ;{ }^{13} \mathrm{C} \mathrm{NMR}\left(\mathrm{CDCl}_{3}\right)$ 164.66, 157.84, 151.67, $145.57,140.58,134.13,129.17,124.16,117.53,114.49,87.40,55.43,50.16$.

2,3-Dihydro-3-methoxy-2,3-diphenyl-1H-pyrrolo[3,4-c]pyridine-1-one (4c). (50\%), (silica gel, eluent dichloroethane : ethyl acetate - 1:1, $\mathrm{R}_{\mathrm{f}}$ 0.4), m. p. 102-105 ${ }^{\circ} \mathrm{C}$ (heptane); (Found: $\mathrm{C}$, 75.8; H, 5.1; N, 8.8. Calc. for $\mathrm{C}_{20} \mathrm{H}_{16} \mathrm{~N}_{2} \mathrm{O}_{2}$ : C, 75.93; H, 5.10; N, 8.85\%); IR (KBr) $1710 \mathrm{~cm}^{-1}$ $(\mathrm{C}=\mathrm{O}) ;{ }^{1} \mathrm{H}$ NMR $\left(\mathrm{CDCl}_{3}\right) 8.87(1 \mathrm{H}, \mathrm{d}, \mathrm{J}=4.8 \mathrm{~Hz}, 6-\mathrm{H}), 8.59(1 \mathrm{H}, \mathrm{s}, 4-\mathrm{H}), 7.90-7.40$ (1H, two overlapping $\mathrm{d}, \mathrm{J}=4.8 \mathrm{~Hz}, 7-\mathrm{H}), 7.55-7.08(10 \mathrm{H}, \mathrm{m}, \mathrm{Ph}-\mathrm{H}), 3.21(3 \mathrm{H}, \mathrm{s}, \mathrm{OMe})$; ${ }^{13} \mathrm{C} \mathrm{NMR}$ $\left(\mathrm{CDCl}_{3}\right)$ 165.76, 151.19, 145.63, 139.23, 139.00, 137.58, 135.59, 128.80, 128.65, 128.34, $126.44,126.05,124.20,117.45,97.09,50.78$.

\section{Acknowledgements}

This work was supported by Grant-in-Aid for Scientific Research from the University of Łódź. 


\section{References}

1. (a) Botero Cid, H. M.; Tränkle, C.; Baumann, K.; Pick, R.; Mies-Klomfass, E.; Kostenis, E.; Mohr, K.; Holzgrabe, U. J. Med. Chem. 2000, 43, 2155. (b) Danheiser, R. L.; Kwasigroch, C. A.; Tsai, Y.-M. J. Am. Chem. Soc. 1985, 107, 7233.

2. (a) Dunet, A.; Willemart, A. Bull. Soc. Chim. France 1948, 887. (b) Eberle, M. K.; Houlihan, W. J.; Schirm, P. J. Org. Chem. 1973, 38, 3872. (c) Scartoni, V.; Morelli, I.; Marsili, A.; Catalano, S. J. Chem. Soc., Perkin Trans. 1, 1977, 2332. (d) Kreher, R. P.; Konrad, M. R. Chem.-Ztg. 1986, 110, 363. (e) Uozumi, Y.; Mori, E.; Mori, M.; Shibasaki, M. J. Organomet. Chem. 1990, 399, 93. (f) Broquet, C.; Genet, J.-P. C. R. Acad. Sci. Ser. C 1967, $265,117$.

3. (a) Goto, T.; Utsunomiya, S.; Aiba, H.; Hayasaka, H.; Endo, M.; Watanabe, R.; Ishizaki, T.; Sato, R.; Saito, M. Bull. Chem. Soc. Jpn., 1991, 64, 1901. (b) Hoarau, C.; Couture, A.; Cornet, H.; Deniau, E.; Grandclaudon, P. J. Org. Chem., 2001, 66, 8064. (c) Rys, V.; Couture, A.; Deniau, E.; Lebrun, S.; Grandclaudon, P. Synlett, 2004, 12, 2233. (d) Hamprecht, G.; Zimmermann, N.; Weiß, T. D. Eur. J. Org. Chem. 2004, 3551.

4. (a) Flitsch, W. Ber. 1970, 103, 3205. (b) Valters, R. E. Usp. Khim. 1973, 42, 1060. (c) Nashio, T.; Yamamoto, H. J. Heterocycl. Chem. 1995, 32, 883.

5. (a) Epsztajn, J.; Jóźwiak, A.; Szcześniak, A. K. Synth. Commun. 1994, 24, 1789. (b) Epsztajn, J.; Jóźwiak, A.; Czech, K.; Szcześniak, A. K. Monatsh. Chem. 1990, 121, 909. (c) Epsztajn, J.; Grzelak, R.; Jóźwiak, A. Synthesis 1996, 1212.

6. Vogel, A. I. Vogel's Textbook of Practical Organic Chemistry, Longman Scientific \& Technical: London, 1989; pp 409, 410. 